\title{
Lipocalin 2 deactivates macrophages and worsens pneumococcal pneumonia outcomes
}

\author{
Joanna M. Warszawska,,,2 Riem Gawish,,1,2 Omar Sharif,1,2 Stefanie Sigel,1,2 Bianca Doninger,,1,2 \\ Karin Lakovits, ${ }^{2}$ Ildiko Mesteri, ${ }^{3}$ Manfred Nairz, ${ }^{4}$ Louis Boon, ${ }^{5}$ Alexander Spiel, 6 \\ Valentin Fuhrmann, ${ }^{6}$ Birgit Strobl, ${ }^{7}$ Mathias Müller, ${ }^{7}$ Peter Schenk, ${ }^{6}$ \\ Günter Weiss, ${ }^{4}$ and Sylvia Knapp ${ }^{1,2}$
}

\begin{abstract}
${ }^{1}$ Research Center for Molecular Medicine of the Austrian Academy of Sciences (CeMM), Vienna, Austria. ${ }^{2}$ Department of Medicine 1, Laboratory of Infection Biology, and ${ }^{3}$ Department of Pathology, Medical University of Vienna, Vienna, Austria. ${ }^{4}$ Department of Medicine 6 , Clinical Immunology and Infectious Diseases, Medical University of Innsbruck, Innsbruck, Austria. ${ }^{5}$ Bioceros, Utrecht, The Netherlands. ${ }^{6}$ Department of Medicine 3, Medical University of Vienna, Vienna, Austria. ${ }^{7}$ Institute of Animal Breeding and Genetics, University of Veterinary Medicine Vienna, Vienna, Austria.
\end{abstract}

\begin{abstract}
Macrophages play a key role in responding to pathogens and initiate an inflammatory response to combat microbe multiplication. Deactivation of macrophages facilitates resolution of the inflammatory response. Deactivated macrophages are characterized by an immunosuppressive phenotype, but the lack of unique markers that can reliably identify these cells explains the poorly defined biological role of this macrophage subset. We identified lipocalin 2 (LCN2) as both a marker of deactivated macrophages and a macrophage deactivator. We show that LCN2 attenuated the early inflammatory response and impaired bacterial clearance, leading to impaired survival of mice suffering from pneumococcal pneumonia. LCN2 induced IL-10 formation by macrophages, skewing macrophage polarization in a STAT3-dependent manner. Pulmonary LCN2 levels were tremendously elevated during bacterial pneumonia in humans, and high LCN2 levels were indicative of a detrimental outcome from pneumonia with Gram-positive bacteria. Our data emphasize the importance of macrophage deactivation for the outcome of pneumococcal infections and highlight the role of LCN2 and IL-10 as determinants of macrophage performance in the respiratory tract.
\end{abstract}

\section{Introduction}

Macrophages are innate immune cells involved in the maintenance of tissue homeostasis and in the pathogenesis of diverse conditions such as metabolic, allergic, fibrotic, autoimmune, and neoplastic diseases (1). Macrophages are well known to play decisive roles during infections, as they express a number of pattern recognition receptors that enable them to sense pathogens (2). In doing so, macrophages initiate an inflammatory response to combat microbe multiplication, which is followed by a deactivation process that facilitates the resolution of inflammation after microbial elimination. The basis for these pleiotropic functions of macrophages is their enormous phenotypic and functional plasticity, also referred to as "macrophage polarization."

Bacterial pneumonia is an excellent model to study the poorly understood plasticity of macrophages in vivo, where macrophages must rapidly adjust to an environment of constantly changing stimuli (3). In the setting of the clinically important model of pneumococcal pneumonia, it is well established that an initial proinflammatory response facilitates the clearance of pathogens and that the subsequent antiinflammatory response limits tissue damage by phagocytosis of apoptotic neutrophils and cell debris (4-7). Pulmonary macrophages are importantly involved in shaping the inflammatory response upon infection, although the precise pathways that govern these steps in vivo are incompletely understood.

In vitro, macrophages polarize to proinflammatory, classically activated M1 macrophages in the presence of IFN- $\gamma$ and LPS, and to alternatively activated, antiinflammatory M2 macrophages in the presence of IL-4 and/or IL-13 (2, 8, 9). The field of macrophage

Conflict of interest: The authors have declared that no conflict of interest exists. Citation for this article: J Clin Invest. 2013;123(8):3363-3372. doi:10.1172/JCI67911. polarization has expanded substantially over the past years, further reflecting the importance and relevance of different macrophage populations. As such, further characterizations of alternative phenotypes resulted in the discovery of novel subsets, such as regulatory, deactivated, and tumor-associated macrophages (2, 9-11). Deactivated macrophages constitute a remarkably heterogeneous group that arises upon stimulation with TLR ligands in combination with glucocorticoids, IL-10, apoptotic cells (ACs), or prostaglandins (2, 9). Deactivated macrophages are characterized by the downregulation of proinflammatory mediators and the upregulation of antiinflammatory cytokines such as IL-10 (10). While macrophage activation has been studied extensively over the past years, the process of macrophage deactivation is less well understood.

Lipocalin 2 (LCN2) $(12,13)$, also known as neutrophil gelatinase-associated lipocalin, is a mammalian protein expressed by myeloid and epithelial cells in response to TLR activation during infections $(14,15)$. LCN2 is mainly known as an antimicrobial defense mediator that scavenges a subset of bacterial siderophores, thereby restricting iron acquisition by bacteria such as Escherichia coli $(14,16)$, Salmonella typhimurium (17), Klebsiella pneumoniae (15), or mycobacteria (18). However, Streptococcus pneumoniae, the most prevalent respiratory pathogen, does not depend on siderophores for iron acquisition (19). We and others (20) found that $S$. pneumoniae induced remarkably high LCN2 levels in the respiratory tract. This finding alerted us to the possibility of a siderophore-independent role of LCN2 within the pulmonary compartment. Upon analysis of the published LCN2 promoter (21) we noticed NF- $\mathrm{KB}$ binding sites, but also glucocorticoid response elements and several binding sites for transcription factors critically involved in the polarization of M2 macrophages (22), such as STAT3, CREB, and C/EBP $\beta$. Considering that LCN2 may modu- 

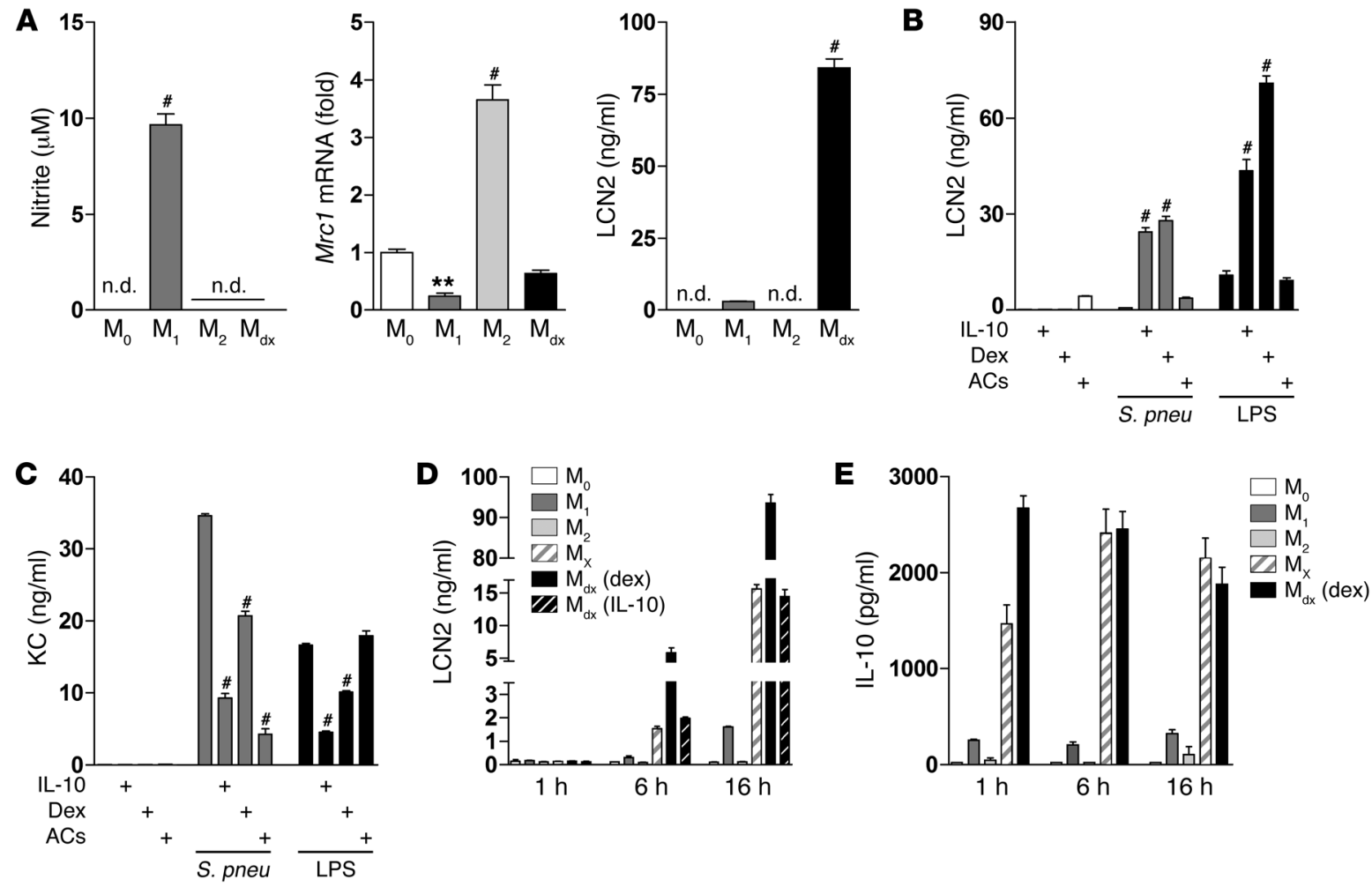

\section{Figure 1}

LCN2 is highly expressed in deactivated macrophages. (A) Primary AMs were polarized with M1 (100 ng/ml LPS, 200 U/ml IFN- $\gamma$ ), M2 (10 ng/ml IL-4 and IL-13), or deactivating ( $\mathrm{M}_{\mathrm{dx}} ; 10 \mathrm{ng} / \mathrm{ml} \mathrm{LPS}, 100 \mu \mathrm{M}$ dexamethasone) stimuli for 16 hours. Nitrite and LCN2 were measured in supernatants using the Griess reagent or ELISA. Mrc1 mRNA levels were determined using qRT-PCR, normalized to HPRT, and expressed as fold change relative to control cells. (B and C) Primary AMs were treated with $4 \times 10^{7} \mathrm{CFU} / \mathrm{ml} \mathrm{S}$. pneumoniae or $10 \mathrm{ng} / \mathrm{ml}$ LPS with or without defined deactivating stimuli (10 ng/ml IL-10, $100 \mu \mathrm{M}$ dexamethasone, or 107/ml ACs) for 16 hours. LCN2 (B) and KC (C) levels were quantified in supernatants using ELISA. (D and E) BMDMs were polarized with M1 $(10 \mathrm{ng} / \mathrm{ml} \mathrm{LPS,} 200 \mathrm{U} / \mathrm{ml} \mathrm{IFN}-\gamma)$ or M2 (10 ng/ml IL-4 and IL-13), activated with $10 \mathrm{ng} / \mathrm{ml}$ LPS (Mx), or deactivated $\left(\mathrm{M}_{\mathrm{dx}}\right)$ with $10 \mathrm{ng} / \mathrm{ml}$ LPS and $100 \mu \mathrm{M}$ dexamethasone (D and $\left.\mathbf{E}\right)$ or $10 \mathrm{ng} / \mathrm{ml} \mathrm{IL-10}$ (D) for 1, 6, and 16 hours. LCN2 (D) and IL-10 (E) levels were assessed in supernatants using ELISA. Data are presented as mean \pm SEM of quadruplicates and are representative of 2 independent experiments. n.d., not detectable. ${ }^{\star \star} P<0.001,{ }^{\#} P<0.0001$ versus M0 (A; ANOVA) or S. pneumoniae or LPS alone (B and C; ANOVA).

late inflammation $(23,24)$, we hypothesized that LCN2 can play a role in macrophage polarization and thereby affect the host defense against pathogens such as $S$. pneumoniae.

\section{Results}

LCN2 is highly expressed in deactivated macrophages. To first determine whether LCN2 expression depends on the macrophage polarization status, we treated primary alveolar macrophages (AMs) with classically activating M1 (LPS and IFN- $\gamma$ ), alternatively activating M2 (IL-4 and IL-13), or deactivating (LPS and dexamethasone) stimuli and quantified LCN2 secretion. These stimuli were specific, as the stable end product of nitric oxide, nitrite, was only detectable in supernatants of M1-stimulated cells (25), and expression of mannose receptor, C type 1 (Mrc1) (8), was exclusively induced in M2-polarized macrophages (Figure 1A). Interestingly, LCN2 was tremendously upregulated upon deactivation with LPS and dexamethasone (Figure 1A). We confirmed these observations using the AM cell line MH-S (Supplemental Figure 1A; supplemental material available online with this article; doi: 10.1172/JCI67911DS1). To test whether LCN2 induction is restricted to glucocorticoid-induced responses or represents a general phenomenon of deactivated macrophages, we treated primary AMs with LPS or S. pneumoniae together with the deactivating stimuli dexamethasone, IL-10, or ACs. The addition of either dexamethasone or IL-10 markedly enhanced the LCN2 release by AMs (Figure 1B) while at the same time decreasing the release of the proinflammatory mediator keratinocyte-derived chemokine (KC) (Figure 1C). ACs solely inhibited the proinflammatory KC response upon $S$. pneumoniae stimulation (Figure $1 \mathrm{C}$ ) but did not affect LCN2 secretion (Figure 1B). We next sought to determine whether LCN2 upregulation was an exclusive feature of deactivated AMs or whether this induction could be extended to other macrophage populations. We therefore repeated the above described experiments using bone marrow-derived macrophages (BMDMs). Dexamethasone enhanced LCN2 release by BMDMs similar to what we found in AMs, whereas IL-10 exerted no effects on LCN2 secretion by BMDMs (Supplemental Figure 1, B and C). To investigate the deactivation of macrophages over time, we polarized BMDMs for 1, 6, and 16 hours and assessed the induction of LCN2 and IL-10, the latter being an established marker for deactivated macrophages (10). This time course showed that IL-10 induction preceded LCN2 releases upon dexamethasone-mediated deactivation of macrophages (Figure 1, D and E). Together, these results indicate that high LCN2 induction is a feature of dexamethasone-deactivated macrophages and a robust marker of IL-10-polarized AMs. 

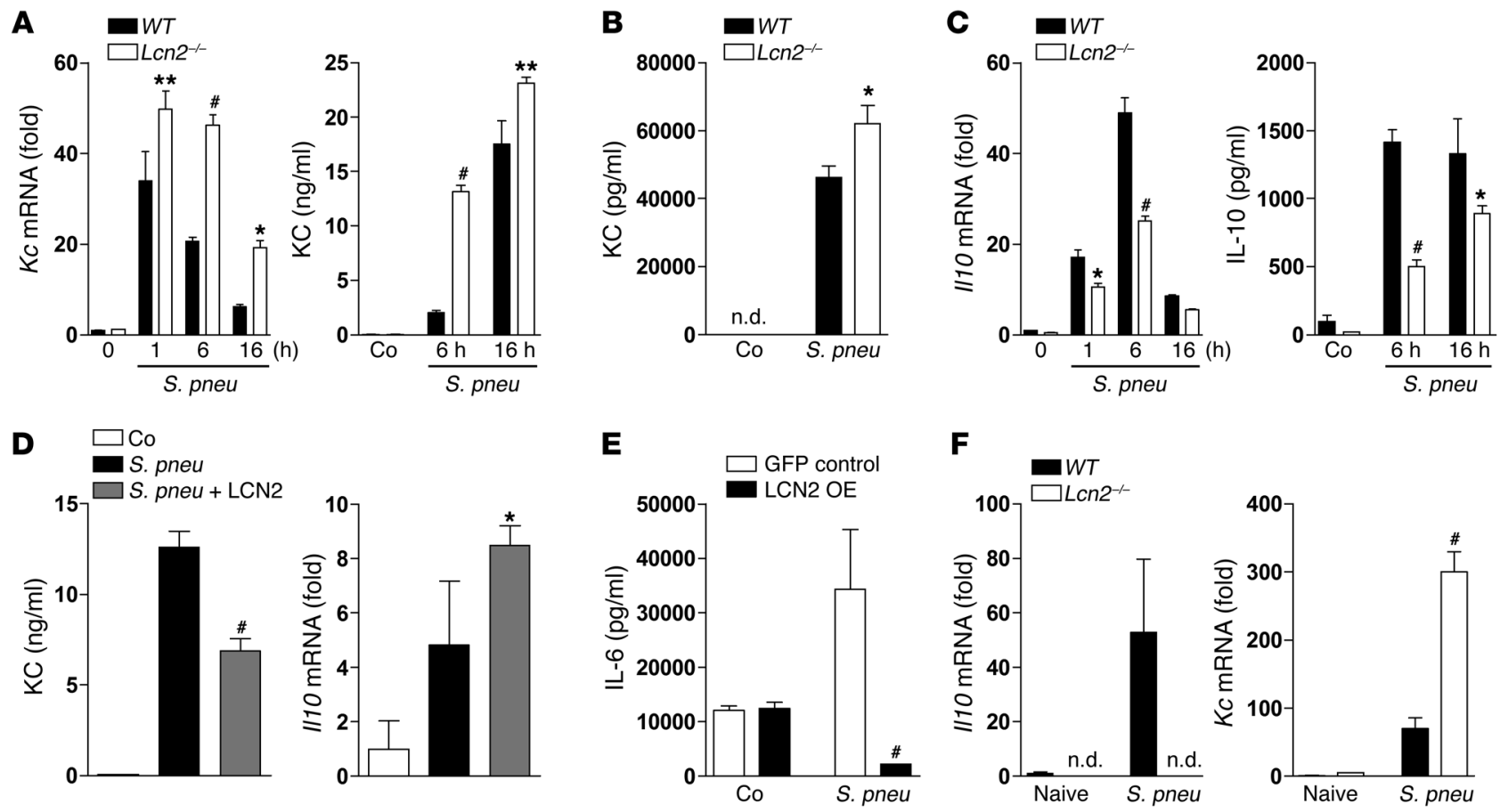

\section{Figure 2}

LCN2 skews macrophages toward a deactivated phenotype. (A and C) WT and Lcn2 ${ }^{-/-}$BMDMs were treated with $4 \times 10^{7}$ CFU/ml S. pneumoniae for 1,6 , and 16 hours. $K c(\mathbf{A})$ and $/ / 10$ (C) expression was measured using quantitative RT-PCR, normalized to HPRT, and expressed as fold change compared with untreated WT cells. KC (A) and IL-10 (C) protein levels were assessed in supernatants using ELISA. (B) KC levels in supernatants of WT and Lcn2-/- AMs stimulated with $4 \times 10^{7} \mathrm{CFU} / \mathrm{ml}$ S. pneumoniae for 16 hours. (D) WT AMs were stimulated with $4 \times 10^{7}$ $\mathrm{CFU} / \mathrm{ml} \mathrm{S}$. pneumoniae $\pm 100 \mathrm{ng} / \mathrm{ml}$ LCN2. KC levels were assessed in supernatants after 16 hours. //10 expression was determined after 1 hour using quantitative RT-PCR, normalized to HPRT, and expressed as fold change compared with control cells. (E) IL-6 release by RAW264.7 cells overexpressing LCN2 or GFP, treated with $4 \times 10^{7} \mathrm{CFU} / \mathrm{ml}$ S. pneumoniae for 16 hours. (F) WT and Lcn2 ${ }^{-/-}$mice were infected with $10^{5} \mathrm{CFU}$ S. pneumoniae. Alveolar cells were isolated after 6 hours by lavage, and $K c$ and $/ / 10$ transcript levels were quantified using quantitative RT-PCR, normalized to HPRT, and expressed as fold change relative to cells from uninfected WT animals. Data are presented as mean \pm SEM of quadruplicates and are representative of 2 independent experiments. ${ }^{*} P<0.05,{ }^{*} P<0.001,{ }^{\sharp} P<0.0001$ versus WT (A-C and F; ANOVA), S. pneumoniae alone (D; $t$ test), or GFP control (E; $t$ test).

LCN2 skews macrophages toward a deactivated phenotype. Having established that LCN2 was highly induced in deactivated macrophages, we next wondered whether LCN2, in analogy to IL-10, itself exerted immunomodulatory effects on these cells. In comparing TLR-induced responses of WT and $L c n 2^{-/-}$BMDMs or AMs, we observed that the absence of LCN2 resulted in a sustained proinflammatory immune response. $L c n 2^{-/-}$macrophages produced significantly more $\mathrm{KC}$, TNF- $\alpha$, and IL-6 in response to $S$. pneumoniae (Figure 2, A and B, and Supplemental Figure 2, A and B) or the TLR2 ligand lipoteichoic acid (LTA) (Supplemental Figure 2C). At the same time, we found the antiinflammatory response impaired, as illustrated by a diminished IL-10 response by $L c n 2^{-/-}$ macrophages (Figure 2C). Since LCN2 modulates intracellular iron homeostasis (17) and because iron negatively affects proinflammatory immune effector pathways in macrophages $(26,27)$, we excluded the possibility that differences in the iron status mediated these effects. In fact, neither the addition of deferoxamine nor of $\mathrm{Fe}(\mathrm{III})$ salts changed the $\mathrm{KC}$ response to $S$. pneumoniae by WT and $L c n 2^{-/-}$BMDMs (Supplemental Figure 2D). We furthermore studied whether the presence of bacterial siderophores is critical for the LCN2-mediated effects on inflammation, and repeated the above-described experiments in the presence or absence of enterobactin. The addition of enterobactin did not change the deacti- vating effects of endogenous LCN2 on macrophages following S. pneumoniae stimulation (Supplemental Figure 2E).

The prominent proinflammatory immune response of $L \mathrm{cn} 2^{-/-}$ macrophages suggested that LCN2 exerted antiinflammatory functions and may itself contribute to the deactivation of macrophages exposed to S. pneumoniae. To next assess the direct effect of LCN2, we treated AMs with S. pneumoniae in the presence or absence of recombinant LCN2. To exclude the possibility of modifying effects of siderophores on cellular iron homeostasis, we used LCN2 expressed in a mouse myeloma cell line, i.e., lacking enterobactin (apo-LCN2). The addition of exogenous apo-LCN2 indeed dampened the proinflammatory response while enhancing the expression of IL-10 (Figure 2D and Supplemental Figure 2F). We verified the dose-dependent suppressive effects of apo-LCN2 using the AM cell line MH-S, stimulated with LTA from S. pneumoniae or S. aureus or with LPS, in the presence of exogenous apo-LCN2 (Supplemental Figure 2, $\mathrm{G}$ and $\mathrm{H})$. Consistent with the idea of LCN2 dampening inflammation, LCN2 overexpression in RAW264.7 macrophages prevented IL-6 induction upon S. pneumoniae challenge (Figure 2E).

To ultimately investigate the potential impact of endogenous LCN2 on pulmonary macrophages in vivo, we infected WT and Lcn $2^{-/-}$mice with S. pneumoniae for 6 hours, isolated alveolar cells (consisting of $88 \%$ AMs and $12 \%$ neutrophils) by lavage and quanti- 

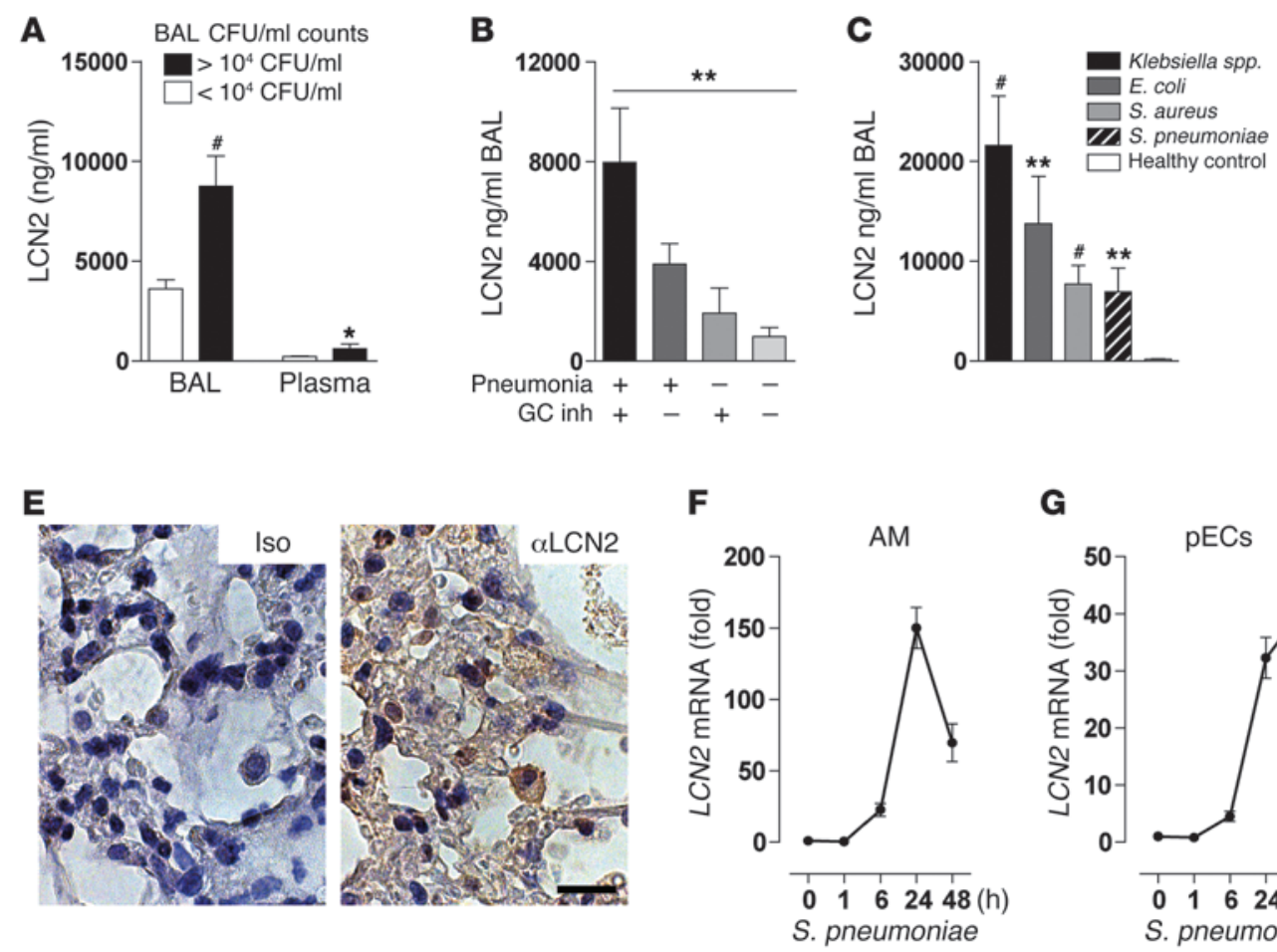

G

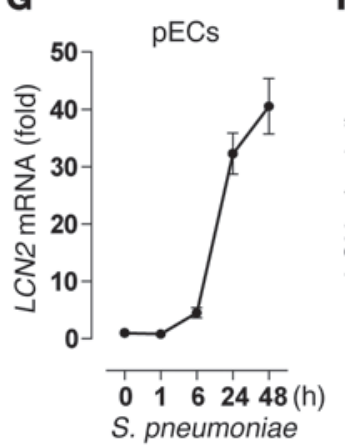

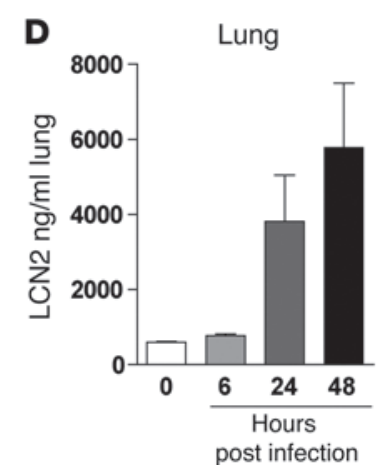

H

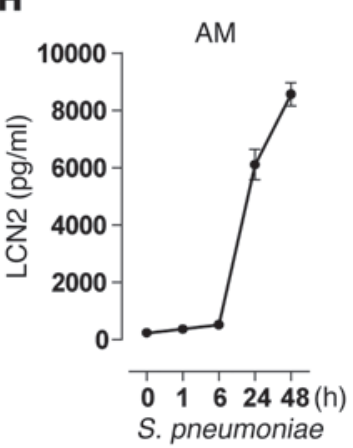

Figure 3

LCN2 is induced during infection in humans and mice. (A-C) LCN2 levels were measured in BAL and plasma of mechanically ventilated patients in the ICU with suspected pneumonia (pulmonary infiltrate in the chest $\mathrm{x}$-ray; $n=64)(\mathbf{A}-\mathbf{C})$ and of control subjects $(n=12)(\mathbf{C})$. LCN2 concentrations were analyzed according to the presence of bacteria $\left(>10^{4} \mathrm{CFU} / \mathrm{ml}\right)$ in BAL $(\mathbf{A})$, according to the presence of pneumonia and history of treatment with inhalative glucocorticoids prior to bronchoscopy (B), and per identified pathogen ( $n=5$ [Klebsiella spp., E. coli]; 6 [S. aureus, S. pneumoniae], 12 [healthy]) (C). (D and E) WT mice were infected with $10^{5}$ CFU S. pneumoniae, and (D) pulmonary LCN2 concentrations were quantified over time. (E) Lungs were stained immunohistochemically using rat IgG2a (left) or anti-LCN2 Ab (right) 48 hours after infection. Scale bar: $20 \mu \mathrm{m}$. (F-H) Primary AMs $(\mathbf{F}$ and $\mathbf{H})$ or primary epithelial cells $(\mathbf{G})$ were treated with $2 \times 10^{7} \mathrm{CFU} / \mathrm{ml}$ S. pneumoniae for the indicated times. (F and $\left.\mathbf{G}\right) \mathbf{L C N 2}$ transcript levels were assessed by quantitative RT-PCR, normalized to HPRT, and expressed as fold change versus baseline. (H) LCN2 release was evaluated in supernatants using ELISA. Data are expressed as mean \pm SEM $(n=8$ [D and E]; 4 [F-H]) and are representative of 2 independent experiments. ${ }^{\star} P<0.05,{ }^{\star \star} P<0.001,{ }^{\#} P<0.0001$ versus patients in the ICU without pneumonia $(\mathbf{A} ; t$ test $)$ or healthy subjects $(\mathbf{C} ;$ ANOVA).

fied KC and IL-10 transcript levels. We found endogenous LCN2 capable of keeping alveolar cells in a deactivated state, as illustrated by increased Il10 and suppressed $K c$ mRNA expression upon S. pneumoniae infection in WT but not $L c n 2^{-/-}$mice (Figure 2F). Collectively, these overexpression, substitution, and deletion experiments illustrate that LCN2 deactivates macrophages in vitro and in vivo.

LCN2 is induced during infection in bumans and mice. The finding that endogenous LCN2 deactivated AM during pneumococcal infection (Figure 2F) encouraged us to examine in more detail the potential function of LCN2 during pneumonia. To begin, we tested the possible induction of pulmonary LCN2, which to our knowledge had not previously been studied in patients with bacterial lung infections. Because we wanted to investigate the systemic and local, pulmonary induction of LCN2, we concentrated on mechanically ventilated patients in an intensive care unit (ICU), from whom alveolar lavage samples were made available during routinely performed bronchoscopies. We found that LCN2 concentrations in BAL and plasma were significantly enhanced in patients with confirmed bacterial pneumonia (pulmonary infiltrates in chest $x$-rays and bacterial CFU counts $>10^{4} / \mathrm{ml}$ BAL; Figure $3 \mathrm{~A}$ ). LCN2 concentrations in BAL were approximately 10-fold higher than in plasma, indicating the local production of LCN2 at the site of infection (Figure 3A). Notably, inhaled glucocorticoid therapy (as part of the acute treatment) increased pulmonary LCN2 levels in patients with confirmed bacterial pneumonia (Figure 3B), suggesting that these potent macrophage-deactivating drugs also induced LCN2 in vivo in humans. Intriguingly, we found increased LCN2 levels not only in patients with pneumonia caused by pathogens producing siderophores that are recognized by LCN2 (such as Klebsiella or E. coli), but also in patients infected with bacteria whose siderophores are not recognized by LCN2 (e.g., S. aureus) or with bacteria that do not utilize siderophores at all (e.g., S. pneumoniae) (Figure 3C). These findings hint at a siderophore-independent role of LCN2 during pulmonary infections.

To examine this potential siderophore-independent role of LCN2, we made use of a murine pneumococcal pneumonia model and first quantified pulmonary LCN2 concentrations upon infection. We observed a steady increase in LCN2 levels starting 6 hours after infection with S. pneumoniae (Figure 3D). We next searched for the cellular origin of LCN2 during pneumococcal infection, and immunohistochemical stainings of lungs revealed LCN2 expression in both hematopoietic and epithelial cells 48 hours after S. pneumoniae infection (Figure 3E). We therefore tested LCN2 levels in primary AMs and primary respiratory epithelial cells treated with $S$. pneumoniae and found more pronounced mRNA and protein expression in 
A

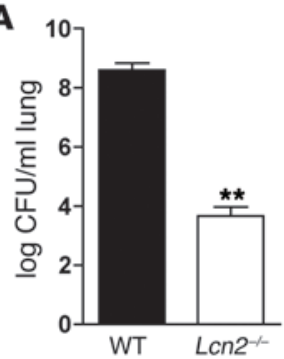

\section{E}

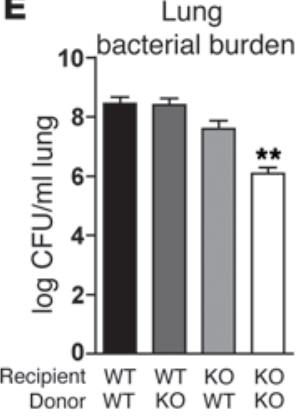

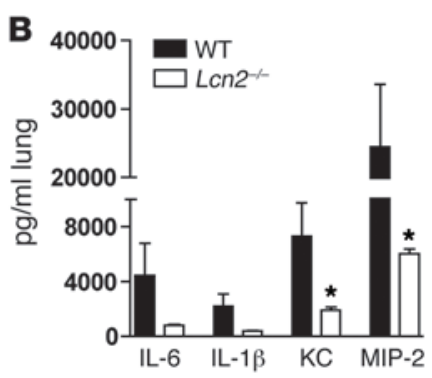

$\mathbf{F}$

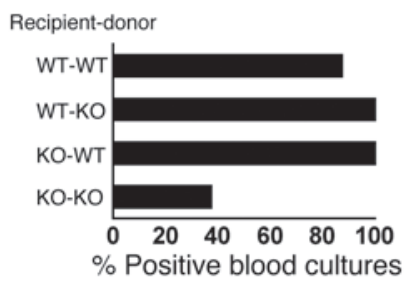

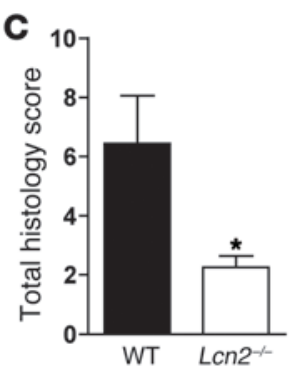
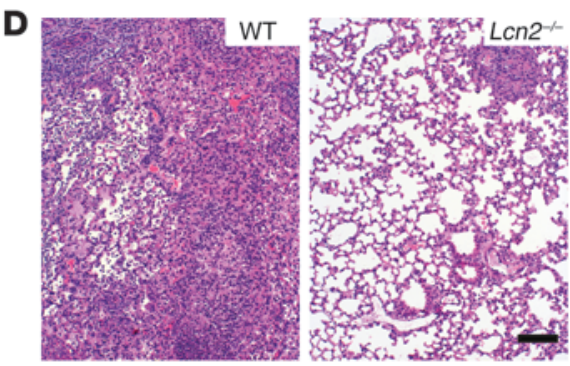

G

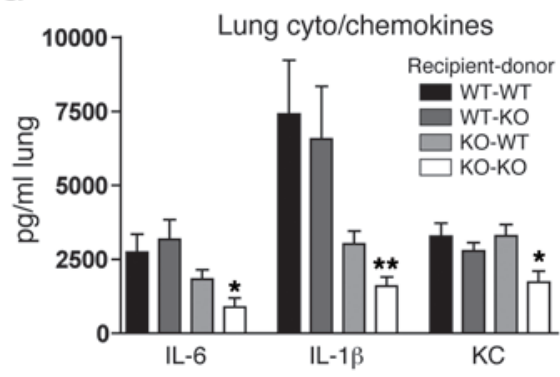

H

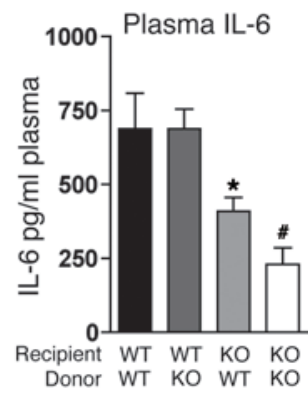

Figure 4

LCN2 impairs bacterial clearance in vivo. (A-D) WT and Lcn2--- mice were intranasally infected with $2 \times 10^{4}$ CFU S. pneumoniae for 48 hours. (A and B) Bacterial CFU counts were enumerated in lungs (A), and cytokine secretion was quantified in lung homogenates using ELISA (B). (C and D) H\&E staining of lung sections. (C) Sections were scored as described in Methods. (D) Representative images. Scale bar: $100 \mu \mathrm{m}$. (E-H) WT mice reconstituted with WT bone marrow (WT-WT), Lcn2 $2^{-1-}$ mice reconstituted with $L c n 2^{-1-}$ bone marrow (KO-KO), and chimeric mice (WT-KO and KO-WT) were infected intranasally with $10^{5} \mathrm{CFU}$ S. pneumoniae. (E and F) Bacterial growth was quantified 48 hours after infection in lung homogenates $(\mathbf{E})$ and blood $(\mathbf{F})$. ( $(\mathbf{G}$ and $\mathbf{H})$ IL-6, IL-1 $\beta$, and KC levels in lungs $(\mathbf{G})$ and IL-6 levels in plasma $(\mathbf{H})$ were quantified using ELISA. Data are presented as mean $\pm \operatorname{SEM}\left(n=8\right.$ per group) and are representative of 2 independent experiments. ${ }^{\star} P<0.05$, ${ }^{\star *} P<0.001$, ${ }^{\#} P<0.0001$ versus WT (A-C; $t$ test) or WT-WT (E, G, and H; ANOVA).

AM than in epithelial cells (Figure 3, F-H). Since polymorphonuclear neutrophils (PMNs) have also been reported to be cellular sources of LCN2 (13), we stained for LCN2 in neutrophils of whole lung cell suspensions obtained from mice infected with $S$. pneumoniae. These assays showed that all neutrophils contained preformed LCN2 in their granules at baseline, and upon progression of pneumonia the proportion of LCN2-positive PMNs gradually decreased (Supplemental Figure 3). These data demonstrate the importance of neutrophil-derived LCN2 and suggest that preformed LCN2 is secreted from neutrophilic granules. Considering the rapid recruitment of PMNs during pneumonia, early increments in lung LCN2 most likely originated from neutrophils. Taken together, these results indicate that pulmonary LCN2 is highly induced in the course of bacterial pneumonia in both mice and humans.

LCN2 impairs bacterial clearance in vivo. Having found LCN2 to exert antiinflammatory effects and to be strongly induced during pneumonia, we next explored the biological function of LCN2 during pneumococcal pneumonia and analyzed the antibacterial response in WT and $L c n 2^{-/-}$mice. First we studied a late time point, at which the efficacy of antibacterial effector mechanisms could be appreciated by the decline in bacterial counts. By doing so, we observed significantly improved bacterial clearance in $\operatorname{Lcn} 2^{-/-}$mice compared with that in WT animals after 48 hours of infection, indicating an improved antibacterial response in the absence of LCN2 (Figure 4A). As a consequence of the reduced bacterial burden in $\mathrm{Lcn} 2^{-/-}$mice, we found an accelerated resolution of inflammation as evidenced by lower levels of inflammatory cytokines in the lung (Figure 4B) and by less severe signs of lung inflammation (Figure 4, C and D). We then sought to determine the respective contribution of myeloid and epithelial cells to the observed phenotype and generated bone marrow-chimeric mice in order to subject them to S. pneumoniae for 48 hours. Although we observed a tendency toward improved bacterial clearance in chimeric $\operatorname{Lcn} 2^{-/-}$mice that had been reconstituted with WT bone marrow (KO-WT mice), only mice completely lacking LCN2 in both the hematopoietic and radioresistant epithelial compartment (KO-KO mice) displayed significantly lower bacterial counts in their lungs after 48 hours (Figure 4E). As a consequence, bacterial dissemination (Figure 4F) as well as pulmonary (Figure 4G) and systemic cytokine formation (Figure $4 \mathrm{H}$ ) were most robustly reduced in $\mathrm{KO}-\mathrm{KO}$ animals. Together, LCN2 produced by both epithelial and myeloid cells was required to impair the host defense against $S$. pneumoniae in vivo.

LCN2 dampens the early immune response. In order to elucidate the mechanism underlying this improved host defense in the absence of LCN2, we evaluated the early (6 hours) immune response to S. pneumoniae in mice, as this early time point resembles the induction phase of inflammation that in turn determines the efficacy of the antibacterial response later on. After infecting WT and $\mathrm{Lcn} 2^{-/-}$ animals with $S$. pneumoniae for 6 hours, we detected a significantly increased release of proinflammatory cytokines and chemokines in $\mathrm{Lcn} 2^{-/-}$animals (Figure 5A). The neutrophil chemoattractant $\mathrm{KC}$ was most impressively increased in $\mathrm{Lcn} 2^{-/-}$mice, resulting in a consecutively enhanced influx of neutrophils (Figure 5B). Since LCN2 has been reported to affect apoptosis (28), we excluded 

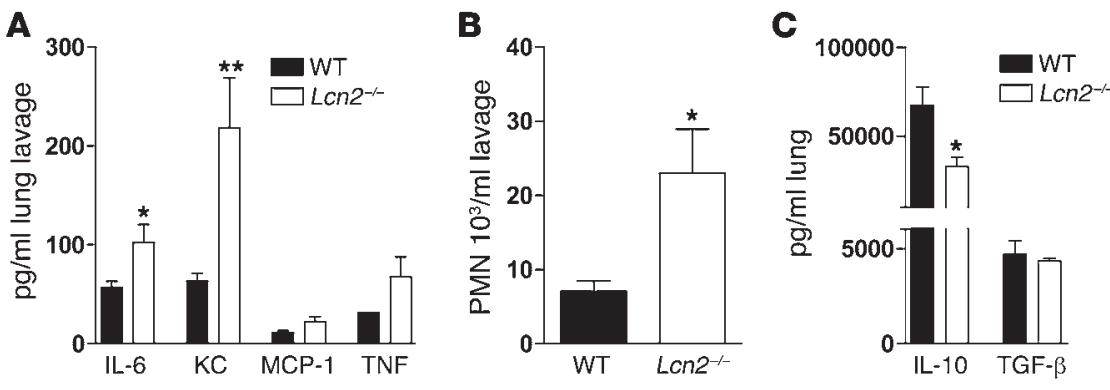

\section{Figure 5}

LCN2 dampens the early immune response. (A-C) WT and $L c n 2^{-/-}$mice were intranasally infected with $3 \times 10^{4}$ CFU S. pneumoniae for 6 hours. (A and C) Secretion of IL-6, KC, MCP-1, and TNF- $\alpha$ in BAL (A) and IL-10 and TGF- $\beta$ in lungs (C) was quantified using ELISA. (B) Neutrophil influx was determined using differential cell counts of cytospin preparations. Data are presented as mean \pm SEM ( $n=8$ per group) and are representative of 2 independent experiments. ${ }^{\star} P<0.05,{ }^{\star \star} P<0.001$ versus WT $(t$ test).

the possibility that increased neutrophil numbers in the BAL of $L c n 2^{-/-}$mice resulted from altered rates of apoptosis (Supplemental Figure 4, A-C) or from a priori differences in white blood cell counts between WT and gene-deficient animals (Supplemental Figure 4D). Interestingly, the antiinflammatory mediator IL-10, but not TGF- $\beta$, was significantly decreased in $L c n 2^{-/-}$as compared with WT mice (Figure 5C). Hence, these in vivo data support our hypothesis of LCN2 being a deactivator of macrophages (Figure 2), as we found LCN2 to dampen the early immune response upon $S$. pneumoniae infection in mice. The resulting delay in the attraction of neutrophils to the site of infection in turn can explain the subsequently impaired bacterial clearance in WT mice (Figure 4A).

LCN2 impairs bacterial clearance in an IL-10-dependent manner. Having observed increased IL-10 levels upon S. pneumoniae stimulation in the presence of LCN2 in vitro and in vivo (Figure 2, C, D, and $\mathrm{F}$, and Figure 5C), we hypothesized that the antiinflammatory properties of LCN2 might depend on the induction of IL-10. To test this hypothesis, we inhibited IL-10 activity using a blocking antibody before treating BMDMs with S. pneumoniae and LCN2. Indeed, IL-10 neutralization completely abolished the inhibitory effects of LCN2 (Figure 6A), confirming that LCN2 required IL-10 to suppress $\mathrm{KC}$ releases. In agreement with this, macrophages deficient in STAT3, the major downstream transcription factor of IL-10, did not display reduced $\mathrm{KC}$ releases in the presence of exogenous LCN2 (Figure 6B). Having established that LCN2 dampens inflammation in a largely IL-10-dependent manner, we next wondered whether LCN2 directly affected transcription of Il10 mRNA. Using RAW264.7 macrophages stably overexpressing LCN2, we discovered enhanced baseline Il10 promoter activity that was significantly augmented following stimulation with $S$. pneumoniae (Figure 6C), indicating that LCN2 enhanced Il10 transcription, especially in the presence of bacteria. In accordance, BMDMs treated with exogenous LCN2 in the absence of bacteria did not induce IL-10 release, whereas LCN2 enhanced the production of IL-10 in a dose-dependent manner when concomitantly stimulated with S. pneumoniae (Figure 6D). To summarize, we found that LCN2 deactivates macrophages through an IL-10/STAT3-dependent mechanism via the induction of IL-10 in the presence of bacteria.

Because the in vitro antiinflammatory properties of LCN2 were mediated via IL-10 (Figure 6, A and B), and considering the detrimental effects of IL-10 during pneumococcal pneumonia the resolution of inflammation (Figure 6G).

$(29,30)$, we hypothesized that LCN2 might dampen inflammation and thus impair the host defense to $S$. pneumoniae via induction of IL-10 in vivo. To examine this idea, we blocked IL-10 in WT and $L \mathrm{cn}^{-{ }^{--}}$animals that were infected with $S$. pneumoniae for 48 hours. Consistent with previous data (Figure 4A), Lcn $2^{-/-}$mice treated with isotype Ab cleared $S$. pneumoniae from their lungs more efficiently than their WT counterparts (Figure 6E). In agreement with earlier reports (29), blocking of IL-10 decreased bacterial counts in WT animals, while no significant change was observed in $\mathrm{Lcn} 2^{-/-}$mice. Of note, application of the anti-IL-10 Ab completely abolished the differences in pulmonary and systemic bacterial clearance between WT and $L c n 2^{-/-}$ animals (Figure 6, E and F) and accelerated In conclusion, our results confirm that LCN2 impairs bacterial clearance in a largely IL-10-dependent manner in vivo.

LCN2 exerts detrimental effects during pneumococcal pneumonia in mice and humans. The improved bacterial clearance in the absence of LCN2 suggested that LCN2 might negatively affect the outcome of pneumococcal pneumonia. To test this hypothesis we infected WT and $L c n 2^{-1-}$ mice with S. pneumoniae and monitored survival over 7 days. While $70 \%$ of $L c n 2^{-1-}$ mice recovered, more than $90 \%$ of WT mice succumbed to the infection (Figure 7A). In accordance with these murine data, high LCN2 levels were indicative of an adverse clinical outcome in the BAL of patients in the ICU who were suffering from pneumonia caused by Gram-positive bacteria (that were either siderophore independent or that produced siderophores not recognized by LCN2; Supplemental Table 1) but not by Gram-negative bacteria (which predominantly depended on LCN2-compatible siderophores; Figure 7B). Of note, none of the nonsurvivors suffering from pneumonia with Gram-positive bacteria received inhaled glucocorticoids. Taken together, these data strengthen the hypothesis that LCN2 exerts detrimental effects during pneumococcal pneumonia in mice and indicate that enhanced pulmonary LCN2 levels in humans suffering from pneumonia with Gram-positive bacteria are predictive of a poor outcome.

\section{Discussion}

Deactivated macrophages are characterized by downregulation of proinflammatory cytokines and upregulation of IL-10 (10). Compared with M1 or M2 polarized cells, the precise functions of deactivated macrophages are still poorly understood, and even less is known about their biological role during infections. Here, we identified LCN2 as a novel marker and mediator of deactivated macrophages in mice. Our studies illustrate that the presence of LCN2 was associated with macrophage deactivation in the lungs, which resulted in an impaired immune response to the clinically important pathogen S. pneumoniae. Mechanistically, we found IL-10 to mediate the antiinflammatory effects of LCN2 upon infection.

Among the macrophage subsets we studied, the highest LCN2 levels were released by AMs, and AMs were the only cells in which IL-10 further enhanced the synthesis of LCN2. Since AMs are the only macrophage subset that is constantly exposed to environmental microbes, tight control of its activation status is required. Our data suggest an essential role for LCN2, which is consistent 

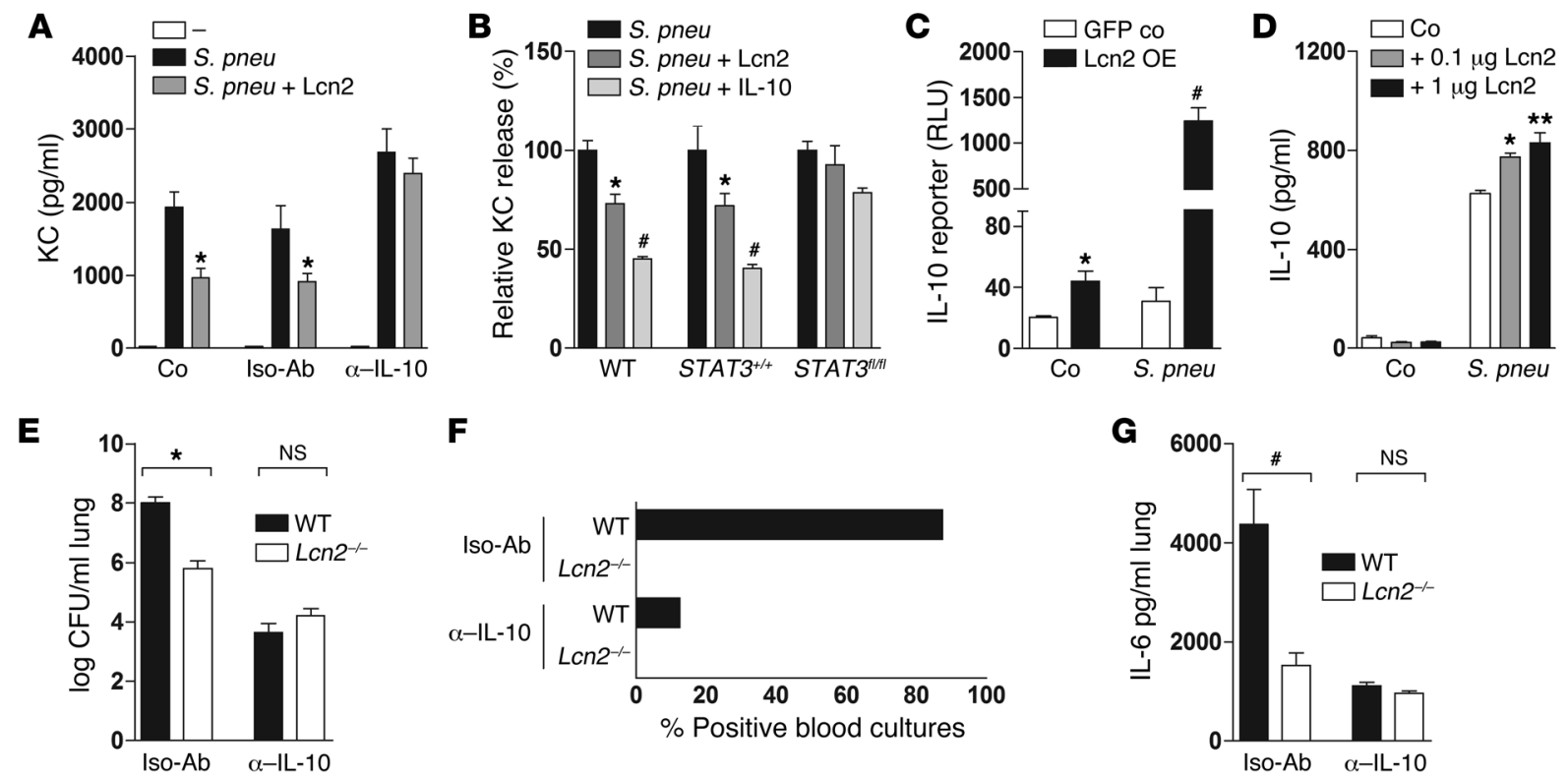

$\mathbf{F}$

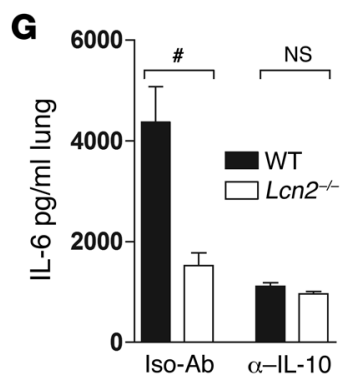

Figure 6

LCN2 impairs bacterial clearance in an IL-10-dependent manner. (A) KC levels in supernatants of WT BMDMs stimulated with $4 \times 10^{7}$ CFU/ml S. pneumoniae with or without $100 \mathrm{ng} / \mathrm{ml} \mathrm{LCN2}$ and with or without $10 \mu \mathrm{g} / \mathrm{ml}$ anti-IL-10 or isotype control antibody for 6 hours. (B) Normalized KC secretion from WT, STAT3 ${ }^{+/+}$, and STAT3 fl/fl BMDMs treated with $4 \times 10^{7} \mathrm{CFU} / \mathrm{ml}$ S. pneumoniae plus LCN2 (100 ng/ml) or IL-10 (10 ng/ml) for 6 hours. (C) RAW264.7 overexpressing LCN2 or GFP, transfected with an IL-10 reporter and stimulated with $4 \times 10^{7} \mathrm{CFU} / \mathrm{ml}$ S. pneumoniae for 24 hours. Reporter gene activity was measured in cell lysates and normalized to Renilla. (D) WT BMDMs were treated with $4 \times 10^{7}$ CFU/ml S. pneumoniae plus LCN2 $(0.1 \mu \mathrm{g} / \mathrm{ml}$ and $1 \mu \mathrm{g} / \mathrm{ml})$ for 6 hours. IL-10 release was measured in supernatants using ELISA. (E-G) WT and Lcn2 ${ }^{-/-}$ mice were intranasally infected with $10^{5} \mathrm{CFU}$ S. pneumoniae, followed by intranasal administration of $150 \mu \mathrm{g}$ anti-IL-10 mAb or isotype control Ab. (E and F) Bacterial counts in lung (E) and blood (F) were quantified 48 hours after infection. (G) IL-6 secretions in lung homogenates were determined using ELISA. Data are presented as mean \pm SEM $\left(n=4[\mathbf{A}-\mathbf{D}] ; 8\right.$ [E-G] per group). ${ }^{\star} P<0.05,{ }^{\star \star} P<0.001,{ }^{\#} P<0.0001$ versus S. pneumoniae alone (A, B, and D; ANOVA), GFP control (C; $t$ test), or WT (E and G; ANOVA).

with the homeostatic and antiinflammatory role of AMs (7) and the important regulatory function of IL-10 in the lungs (31). Although our findings indicate that LCN2 preferentially affects the function of AMs, it is tempting to speculate that other antiinflammatory macrophage subsets might also respond to IL-10/ LCN2 induction. Potential candidates include intestinal myeloid regulatory cells (Mregs), given the importance of IL-10 in intestinal homeostasis (32), and also tumor-associated macrophages, since LCN2 was found to be significantly elevated in the plasma of tumor-bearing mice (33). In fact, while this manuscript was in preparation, Jung et al. reported that IL-10 induced LCN2 in human macrophages (34). By keeping breast cancer cells in either conditioned medium from IL-10-stimulated macrophages or medium containing exogenous LCN2, an enhanced growth rate of tumor cells was observed. This led the authors to argue that IL-10induced LCN2 might play a role in tumor-associated macrophage biology (34). These data strengthen our observation of LCN2 as a mediator of deactivated macrophages and extend these findings to human cells. In contrast to the findings of Jung et al. (34), we only identified LCN2 induction following stimulation of macrophages with IL-10 in the presence of bacterial TLR ligands, while IL-10 alone did not induce LCN2. In addition to IL-10, we established dexamethasone to be a major inducer of LCN2-associated macrophage deactivation. In support of our results, an expression profiling study done with murine splenic dendritic cells revealed a strong synergistic effect of dexamethasone and LPS on LCN2 expression (35). The interesting fact that inhalative glucocorti- coids enhanced LCN2 levels in BAL from patients with pneumonia extends these findings to humans, as it supports the idea that pulmonary macrophages also express LCN2 in humans. Based on our data and published reports, we postulate that LCN2 secretion is specifically restricted to IL-10 or dexamethasone-associated macrophage deactivation. In support of this notion, published reports illustrate that LCN2 is not induced in M1- or M2-polarized macrophages (36), and is even downregulated in regulatory macrophages that were generated in the presence of immune complexes (37).

Besides the interference with siderophore-dependent bacterial iron acquisition $(14,38)$, LCN2 was shown to influence cellular processes such as mammalian iron homeostasis $(17,39)$, apoptosis $(28,40)$, or inflammatory responses $(24,41)$. In accordance with our data, Zhang et al. reported that LCN2 reduced the LPStriggered induction of cytokines by RAW264.7 macrophages (24). During completion of this manuscript, another group reported enhanced levels of LPS-induced proinflammatory cytokines secreted by LCN2-deficient peritoneal macrophages and BMDMs as compared with WT cells (42). However, in contrast to our data, this report also showed increased IL-10 releases by $\mathrm{Lcn} 2^{-/-}$peritoneal macrophages, which might be due to differences specific to macrophage subset or the fact that we focused on S. pneumoniae. In contrast to observations in macrophages, LCN2 was found to augment IL-8 releases by human lung epithelial cells and neutrophil influx when triggered by the bacterial siderophore (aferric) enterobactin $(23,41)$. We did not observe any proinflammatory effect when incubating murine macrophages with aferric enterobactin 

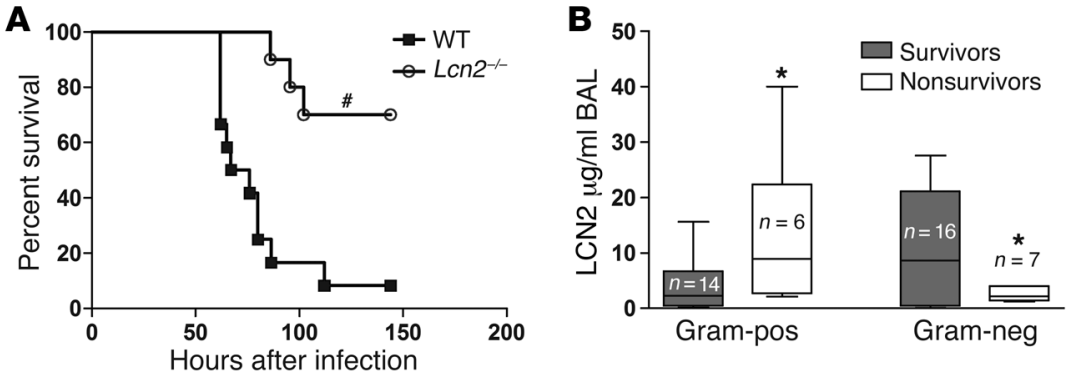

\section{Figure 7}

LCN2 is detrimental during pneumococcal pneumonia in mice and humans. (A) WT and LCn2 ${ }^{-1-}$ mice were infected with $4 \times 10^{4}$ CFU S. pneumoniae and monitored for survival ( $n=12$ per group). ${ }^{*} P<0.0001$ versus WT (log-rank). (B) LCN2 levels in BAL of ICU patients with microbiologically confirmed bacterial pneumonia were analyzed according to the causative pathogen: Gram-positive $(n=20)$ or Gram-negative $(n=23)$ pneumonia. Data are expressed as box-and-whiskers plot. ${ }^{*} P<0.05$ versus survivors $(t$ test).

roids can further enhance LCN2 formation and macrophage deactivation, which is undesirable for the clearance of viable bacteria. Since we found that inhaled corticosteroids enhanced LCN2 levels in patients in the ICU with bacterial pneumonia, the use of these drugs might be harmful during severe infections with pathogens that do not produce siderophores recognizable by LCN2. Thus, LCN2 appears to play a significant role in the course of $S$. pneumoniae pneumonia, and evaluating LCN2 in human BAL may provide significant information on the course of the infection or the necessity to adjust therapy.

\section{Methods}

Human subjects. 64 intubated and mechanically ventilated patients in the ICU with suspected pneumonia, defined by the presence of new infiltrates in the chest $\mathrm{x}$-ray, were examined (21 female, 43 male; age $56 \pm 17$

in the presence of endogenous LCN2 (data not shown). In conjunction with our findings of an antiinflammatory role of LCN2 described here, these reports suggest that LCN2 exerts proinflammatory effects only in the presence of bacteria that release enterobactin, but not in response to bacteria that lack siderophores, such as $S$. pneumoniae. The idea that LCN2 modulates inflammation and scavenges ferric siderophores might explain why the upper respiratory tract is protected from colonization with bacteria releasing enterobactin and is in agreement with the recent observation that the lung microbiome is indeed constituted predominantly of bacteria lacking Lcn2-compatible siderophores (43). The presence of pulmonary LCN2 per se could concurrently explain the dominance of LCN2-resistant bacteria such as S. pneumoniae or Haemophilus influenzae as causative pathogens of pneumonia. Klebsiella pneumoniae, another relevant lung pathogen that depends on enterobactin-like siderophores, evolved LCN2-resistant strains to evade LCN2-mediated growth inhibition (44).

The question remains as to why host cells induce LCN2 upon infection with siderophore-independent bacteria like S. pneumoniae. Because we found that LCN2 induction followed the initial release of IL-10 by macrophages, we postulate that LCN2 might provide a means to prevent overwhelming inflammation and to promote tissue repair after clearance of infectious pathogens. Neutrophils that released substantial amounts of LCN2 late in infection might additionally contribute to the dampening of the immune response and resolution of inflammation through the proposed mechanism, which is in accordance with the recent finding that neutrophils regulate inflammation (45). This pathway might prove safe in uncomplicated infections and even contribute to resolution, which is supported by a report demonstrating a proregenerative function for LCN2 in a model of renal ischemia-reperfusion injury (46). However, in patients in the ICU with severe pneumonia who require mechanical ventilation, elevated LCN2 levels may be a result of an exaggerated immune response to higher bacterial concentrations. In this setting, elevated LCN2 might deactivate macrophages and in turn prevent efficient bacterial clearance and aggravate the disease. In agreement with this hypothesis, we observed a tendency for a worse outcome during severe pneumonia with Gram-positive bacteria in patients in the ICU with elevated LCN2 levels in BAL, although the number of patients we examined in this pilot study was certainly too low to draw definitive conclusions. Corticoste- years, mean $\pm \mathrm{SD} ; 26$ community-acquired and 38 hospital-acquired pneumonia, of which 21 had suspected ventilator-associated pneumonia). Diagnosis of pneumonia was considered confirmed if microbiological assays revealed $>10^{4} \mathrm{CFU} / \mathrm{ml}$ of potentially pathogenic bacteria in the BAL (these patients are referred to as having pneumonia in Figure 3, A-C). 12 agematched outpatient subjects undergoing diagnostic bronchoscopy for noninfectious causes were included as controls ( 5 female, 7 male; age $58 \pm 15$ years). BAL samples were obtained via bronchoscopy, and EDTA blood was withdrawn from ICU patients before bronchoscopy. BAL and plasma LCN2 levels were quantified using ELISA (R\&D).

Mice. Pathogen-free 8- to 10-week-old female C57BL/6 WT and Lcn2 $2^{-/-}$littermate mice were used for experiments. $L c n 2^{-/-}$mice (14) were provided by S. Akira (University of Osaka, Osaka, Japan), and were backcrossed to a C57BL/6 background for 10 generations. Lys $M^{\mathrm{Cr} / / \mathrm{Cre}}$ mice (47) were bred to STAT3 fox fflox mice (48), which were provided by V. Poli (University of Turin, Turin, Italy).

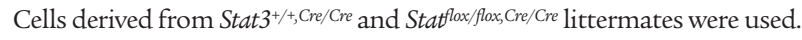

Murine pneumonia model. Pneumococcal pneumonia was induced in mice as described previously $(7,49)$. Briefly, S. pneumoniae serotype 3 (ATCC 6303) was cultured in Todd-Hewitt broth to mid-logarithmic phase, washed twice, and inoculated into anesthetized mice intranasally with $10^{4}-10^{5} \mathrm{CFU} / 50 \mu \mathrm{l}$. Mice were sacrificed with ketamin and xylazine 6, 24, or 48 hours after infection, and blood samples were collected in EDTA-coated tubes. Lungs were harvested and homogenized in sterile saline (Precellys 24; Peqlab) and bacterial growth was quantified by plating 10 -fold serial dilutions on blood agar plates. The remaining lung homogenates were incubated in Greenberger lysis buffer as previously described (50), and supernatants were stored at $-20^{\circ} \mathrm{C}$ until cytokines were assayed. For BAL processing, BAL was performed 6 hours after infection as described previously (51). Cell-free BAL supernatants were stored at $-20^{\circ} \mathrm{C}$ for cytokine measurement. Cell numbers were determined using a hemocytometer, and Giemsa-stained cytospin preparations were used for differential cell counts. For IL-10 blocking in vivo, mice were anesthetized by i.p. injection of ketamine and xylazine and administered $150 \mu \mathrm{g}$ of either anti-IL-10 (clone JES5.2A5, Bioceros) or isotype $\mathrm{Ab}$ (clone GL113, Bioceros) intranasally 15 minutes following S. pneumoniae infection. For histologic examination, lungs were fixed in $10 \%$ formaldehyde and embedded in paraffin. Lung sections $(4 \mu \mathrm{m})$ were stained with H\&E, and the severity of inflammation and pneumonia was analyzed according to a scoring system by a trained pathologist blinded to group assignments. Lungs were examined for the presence of interstitial inflammation, alveolar inflammation, pleuritis, bronchitis, and endothelitis. Each parameter was scored in the range of 0 to 3 points, with 0 repre- 
senting absent, 1 mild, 2 moderate, and 3 severe. Additionally, 1 point each was scored for the presence of pneumonia, edema, and thrombi formation, and 0.5 points for every infiltrate covering $10 \%$ of lung area. The sum of all parameters indicated the total histology score. LCN2 immunostaining was performed on paraffin-embedded lung sections as described previously (51). Briefly, antigen retrieval was performed using a Tris/EDTA, $0.05 \%$ Tween20 buffer at $\mathrm{pH} 8.0$ (Sigma-Aldrich). Lung slides were incubated with rat anti-mouse LCN2 (clone 228418 [R\&D]; coupled to N-hydroxysuccinimide biotin [Calbiochem] in $0.1 \mathrm{M} \mathrm{NaHCO}_{3}$ buffer, $\mathrm{pH} 7.8$ ) or a corresponding isotype control $\mathrm{Ab}$, followed by HRP-conjugated secondary antibody (DakoCytomation) and visualized with 3,3-diaminobenzidine tetrahydrochloride (Vector Laboratories). Nuclei were counterstained with hematoxylin.

Generation of bone marrow-chimeric mice. 9-week-old female WT and Lcn2--mice were irradiated by a single administration of $\gamma$ irradiation ( 9 Gy), and bone marrow was immediately reconstituted by intravenous injection of $2 \times 10^{6}$ freshly isolated bone marrow cells of the same genotype (WT-WT and KO-KO mice) or opposite genotype (chimeric WT-KO and KO-WT mice). 1 mouse of each genotype was left without reconstitution to control for efficiency of irradiation. Mice were kept under sterile conditions for 12 weeks. The proper repopulation of bone marrow-derived cells was confirmed by isolating genomic DNA (gDNA) from blood, AMs, peritoneal macrophages, and bone marrow, followed by genotyping (using PCR). The recipient background was confirmed by genotyping gDNA obtained from tails (Supplemental Figure 5).

Cell culture and stimulations. AMs and BMDMs were isolated as described elsewhere $(6,52)$. Primary cells were cultured in RPMI-1640 (Gibco) containing $1 \%$ penicillin and streptomycin, and $10 \% \mathrm{FCS}$ at $37^{\circ} \mathrm{C}$. MH-S cells (ATCC CRL-2019) were cultured in the same media supplemented with $50 \mathrm{mM}$ 2-mercaptoethanol. For all stimulations, RPMI-1640 containing $3 \%$ FCS was used. Primary epithelial cells were isolated as described previously (53) and cultured in RPMI-1640 containing 1\% penicillin/streptomycin, $2 \% \mathrm{FCS}, 2 \mathrm{mM}$ L-glutamine, $5 \mu \mathrm{g} / \mathrm{ml}$ insulin, $10 \mathrm{nM}$ hydrocortisone, $10 \mathrm{nM} \beta$-estradiol, $100 \mu \mathrm{g} / \mathrm{ml}$ transferrin, and $30 \mathrm{nM}$ sodium selenite (all Sigma-Aldrich). In brief, single-cell lung suspensions were obtained by digesting perfused lungs with dispase and DNase I, followed by several steps of filtration via nylon mesh filters. Enrichment for epithelial cells was achieved by negative selection using plates coated with $\mathrm{Ab}$ against $\mathrm{CD} 45$ and CD16/CD32. IFN- $\gamma$ (PeproTech), LPS from E. coli 055:B5 (Sigma-Aldrich), rmIL-4 (R\&D), rmIL-13 (R\&D), dexamethasone (ratiopharm), rmIL-10 (eBioscience), LTA from S. pneumoniae (TIGR4) (54) and LTA from S. aureus (55), enterobactin-free rmLCN2 produced in a mouse myeloma cell line (R\&D; catalog no. 1857-LC), deferoxamine (Calbiochem), ammonium $\mathrm{Fe}(\mathrm{III})$ citrate (Sigma-Aldrich), and enterobactin (Fe-free) (EMC Microcollections) were used at indicated concentrations for cell stimulations.

Analysis of soluble factors. Mouse LCN2, TNF- $\alpha$, IL-6, KC, IL-1 $\beta$, macrophage inflammatory protein 2 (MIP-2), IL-10, TGF- $\beta 1$, and human LCN2 were measured using specific ELISAs (R\&D Systems or Bender MedSystems for IL-10) according to the manufacturers' instructions. Nitrite levels were estimated using the Griess Reagent System (Promega).

RT-PCR. Messenger RNA was isolated using TRIzol Reagent (Invitrogen) and transcribed into cDNA using iScript cDNA Synthesis Kit (Bio-Rad). Quantitative RT-PCR was performed using iTaq SYBR Green Supermix (BioRad) and the StepOnePlus cycler (Applied Biosystems). Primers for LCN2, MRC1, IL-10, KC, IL-6, and hypoxanthine guanine phosphoribosyl transferase 1 (HPRT) were obtained from Sigma-Aldrich (Supplemental Table 2).

Luciferase gene reporter assays. Stable overexpressing RAW264.7 cells were generated by retroviral transfection. Briefly, the packaging cell line GP-293 HEK (Clontech) was transfected with LCN2 expression plasmid (provided by R.B. Arlinghaus, University of Texas, Houston, Texas, USA) or GFP control plasmids and VSV-G (retroviral vector). RAW 264.7 cells were infected with the virus containing supernatants from HEK cells and successfully transfected, and GFP-expressing cells were sorted using flow cytometry. For more details, see Supplemental Table 1. LCN2-expressing and GFP control cells were then transiently transfected with IL-10 promoter firefly luciferase (56) (pGL2B, -1,538/+64; Addgene) and renilla luciferase (Promega) using the Amaxa Nucleofector Kit (Lonza) according to the manufacturer's instructions. Cells were stimulated 24 hours later with S. pneumoniae, and luminescence was assessed after 24 hours. Data are presented as RLU after correction for Renilla.

Flow cytometry. Lungs were perfused through the heart with PBS, cut into pieces, and placed in RPMI-1640 containing 5\% FCS, collagenase I (Invitrogen), and DNase I (Sigma-Aldrich) for 1 hour at $37^{\circ} \mathrm{C}$. Single-cell suspensions were obtained using a glass homogenizer (Kontes Scientific Glassware/Instruments), followed by passage through $70-\mu \mathrm{m}$ and $40-\mu \mathrm{m}$ cell strainers (BD). Cells were counted and $10^{6} \mathrm{cells} / \mathrm{staining}$ were used. Unspecific binding was blocked using mouse IgG ( $6 \mu \mathrm{g} /$ staining; Invitrogen). Cell surface markers were stained in PBS containing $2 \%$ FCS. The following antibodies were used: Horizon V500 conjugated anti-CD45 (30-F11; BD), FITCconjugated anti-Ly6G (1A8; Biolegend), and Alexa Fluor 700-conjugated anti-CD11b (M1/70; eBioscience). The viability of cells was assessed using Fixable Viability Dye eFluor 780 (eBioscience) according to the manufacturer's instructions. Intracellular stainings were performed using the Fix\&Perm kit (An der Grub Bio-Research) using the following antibodies: anti-LCN2 (228418 [R\&D]; coupled to N-hydroxysuccinimide biotin [Calbiochem] in 0.1 $\mathrm{M} \mathrm{NaHCO}_{3}$ buffer, $\mathrm{pH}$ 7.8), biotin-conjugated rat IgG2a (LO-DNP-16; Caltag), and PE-conjugated streptavidin (eBioscience). For assessment of apoptosis, thymocytes or BAL cells were washed twice with PBS and stained for FITC-conjugated Annexin V and 7-amino-actinomycin D (7-AAD) (both BD) in Annexin V-binding buffer according to the manufacturer's instructions.

Generation of ACs. To generate ACs, the thymi were isolated from 4- to 5-week-old C57BL/6 mice and passed twice through a cell strainer. The cells were treated with $10 \mu \mathrm{M}$ etoposide (Sigma-Aldrich) for 5 hours, and the percentage of ACs was assessed using flow cytometry. The cells were thoroughly washed to remove etoposide before being used for stimulations.

Statistics. Data are expressed as mean \pm SEM. 2-group comparisons were made using an unpaired $t$ test; for more than 2 groups, 1-way ANOVA was used, followed by Bonferroni multiple comparisons test or Kruskal-Wallis test combined with Dunn's multiple comparison test, depending on the distribution of the data (parametric or nonparametric, respectively). Survival data were analyzed using the Kaplan-Meier and log-rank statistic. A $P$ value less than 0.05 was regarded as statistically significant.

Study approval. The human study was approved by the Institutional Review Board of the Medical University of Vienna; written informed consent was obtained from conscious participants prior to enrollment or after regaining consciousness (according to regulations by Austrian law). All animal experiments were approved by the Institutional Review Board of the Medical University of Vienna and the Ministry of Sciences (BMWF66.009/0318-II/10b/2008).

\section{Acknowledgments}

This work was funded in part by a research cluster initiative supported jointly by the Medical University of Vienna and the University of Vienna and by a grant provided by the Austrian Science Fund (FWF; I 289-B09) (to S. Knapp). The clinical part of this work was supported by the Scientific Funds of the Mayor of Vienna (to P. Schenk; project 07100). Part of this work was funded by a research grant by the Austrian Science Fund (FWF; SFB-F28; to B. Strobl and M. Müller). We thank L. Riki Cheever for insightful suggestions and critically reviewing the manuscript. Isabella Haslinger's technical assistance with animal experiments is appreciated. 
Received for publication November 19, 2012, and accepted in revised form May 2, 2013.

Address correspondence to: Sylvia Knapp, CeMM - Research Center for Molecular Medicine of the Austrian Academy of Sciences,
Department of Medicine 1, Laboratory of Infection Biology, Medical University of Vienna, Waehringer Guertel 18-20, Vienna 1090, Austria. Phone: 43.1.40400.5139; Fax: 43.1.40400.5167; E-mail: sylvia.knapp@meduniwien.ac.at.
1. Murray PJ, Wynn TA. Protective and pathogenic functions of macrophage subsets. Nat Rev Immunol. 2011;11(11):723-737.

2. Gordon S. Alternative activation of macrophages. Nat Rev Immunol. 2003;3(1):23-35.

3. Mizgerd JP. Acute lower respiratory tract infection. NEngl J Med. 2008;358(7):716-727.

4. Mitchell TJ. Virulence factors and the pathogenesis of disease caused by Streptococcus pneumoniae. Res Microbiol. 2000;151(6):413-419.

5. van der Poll T, Opal SM. Pathogenesis, treatment, and prevention of pneumococcal pneumonia. Lancet. 2009;374(9700):1543-1556.

6. Lagler $\mathrm{H}$, et al. TREM-1 activation alters the dynamics of pulmonary IRAK-M expression in vivo and improves host defense during pneumococcal pneumonia. J Immunol. 2009;183(3):2027-2036.

7. Knapp S, et al. Alveolar macrophages have a protective antiinflammatory role during murine pneumococcal pneumonia. Am J Respir Crit Care Med. 2003;167(2):171-179.

8. Stein M, Keshav S, Harris N, Gordon S. Interleukin 4 potently enhances murine macrophage mannose receptor activity: a marker of alternative immunologic macrophage activation. J Exp Med. 1992; 176(1):287-292.

9. Mosser DM, Edwards JP. Exploring the full spectrum of macrophage activation. Nat Rev Immunol. 2008;8(12):958-969.

10. Mantovani A, Sica A, Sozzani S, Allavena P, Vecchi $\mathrm{A}$, Locati $\mathrm{M}$. The chemokine system in diverse forms of macrophage activation and polarization. Trends Immunol. 2004;25(12):677-686.

11. Mantovani A, Sozzani S, Locati M, Allavena P, Sica A. Macrophage polarization: tumor-associated macrophages as a paradigm for polarized M2 mononuclear phagocytes. Trends Immunol. 2002;23(11):549-555.

12. Kjeldsen L, Johnsen AH, Sengelov H, Borregaard N. Isolation and primary structure of NGAL, a novel protein associated with human neutrophil gelatinase. J Biol Chem. 1993;268(14):10425-10432.

13. Kjeldsen L, Bainton DF, Sengelov H, Borregaard N. Identification of neutrophil gelatinase-associated lipocalin as a novel matrix protein of specific granules in human neutrophils. Blood. 1994;83(3):799-807.

14. Flo $\mathrm{TH}$, et al. Lipocalin 2 mediates an innate immune response to bacterial infection by sequestrating iron. Nature. 2004;432(7019):917-921.

15. Chan YR, et al. Lipocalin 2 is required for pulmonary host defense against Klebsiella infection. JImmunol. 2009;182(8):4947-4956.

16. Berger $T$, et al. Lipocalin 2-deficient mice exhibit increased sensitivity to Escherichia coli infection but not to ischemia-reperfusion injury. Proc Natl Acad Sci U S A. 2006;103(6):1834-1839.

17. Nairz M, et al. Absence of functional Hfe protects mice from invasive Salmonella enterica serovar Typhimurium infection via induction of lipocalin-2. Blood. 2009;114(17):3642-3651.

18. Saiga $H$, et al. Lipocalin 2-dependent inhibition of mycobacterial growth in alveolar epithelium. J Immunol. 2008;181(12):8521-8527.

19. Tettelin $\mathrm{H}$, et al. Complete genome sequence of a virulent isolate of Streptococcus pneumoniae. Science. 2001;293(5529):498-506.

20. Nelson AL, Barasch JM, Bunte RM, Weiser JN. Bacterial colonization of nasal mucosa induces expression of siderocalin, an iron-sequestering component of innate immunity. Cell Microbiol. 2005; 7(10):1404-1417.
21. Park S, Guo J, Kim D, Cheng JQ. Identification of $24 \mathrm{p} 3$ as a direct target of Foxo3a regulated by interleukin-3 through the phosphoinositide 3-kinase/ Akt pathway. J Biol Chem. 2009;284(4):2187-2193.

22. Lawrence T, Natoli G. Transcriptional regulation of macrophage polarization: enabling diversity with identity. Nat Rev Immunol. 2011;11(11):750-761.

23. Nelson AL, Ratner AJ, Barasch J, Weiser JN. Interleukin- 8 secretion in response to aferric enterobactin is potentiated by siderocalin. Infect Immun. 2007;75(6):3160-3168.

24. Zhang J, Wu Y, Zhang Y, Leroith D, Bernlohr DA, Chen $\mathrm{X}$. The role of lipocalin 2 in the regulation of inflammation in adipocytes and macrophages. $\mathrm{Mol}$ Endocrinol. 2008;22(6):1416-1426.

25 . Ehrt S, et al. Reprogramming of the macrophage transcriptome in response to interferon-gamma and Mycobacterium tuberculosis: signaling roles of nitric oxide synthase-2 and phagocyte oxidase. J Exp Med. 2001;194(8):1123-1140.

26. Weiss G, Werner-Felmayer G, Werner ER, Grunewald $\mathrm{K}$, Wachter $\mathrm{H}$, Hentze MW. Iron regulates nitric oxide synthase activity by controlling nuclear transcription. J Exp Med. 1994;180(3):969-976.

27. Oexle $\mathrm{H}$, et al. Pathways for the regulation of interferon-gamma-inducible genes by iron in human monocytic cells. J Leukoc Biol. 2003;74(2):287-294.

28. Devireddy LR, Teodoro JG, Richard FA, Green MR. Induction of apoptosis by a secreted lipocalin that is transcriptionally regulated by IL-3 deprivation. Science. 2001;293(5531):829-834.

29. van der Poll T, Marchant A, Keogh CV, Goldman $\mathrm{M}$, Lowry SF. Interleukin-10 impairs host defense in murine pneumococcal pneumonia. J Infect Dis. 1996;174(5):994-1000

30. van der Sluijs KF, et al. IL-10 is an important mediator of the enhanced susceptibility to pneumococcal pneumonia after influenza infection. J Immunol. 2004;172(12):7603-7609.

31. Sun J, Madan R, Karp CL, Braciale TJ. Effector $\mathrm{T}$ cells control lung inflammation during acute influenza virus infection by producing IL-10. Nat Med. 2009;15(3):277-284.

32. Kayama H, et al. Intestinal CX3C chemokine receptor 1high (CX3CR1high) myeloid cells prevent T-cell-dependent colitis. Proc Natl Acad Sci U S A. 2012;109(13):5010-5015

33. Whiteaker JR, et al. A targeted proteomics-based pipeline for verification of biomarkers in plasma. Nat Biotechnol. 2011;29(7):625-634

34. Jung $M$, et al. Interleukin-10-induced neutrophil gelatinase-associated lipocalin production in macrophages with consequences for tumor growth. Mol Cell Biol. 2012;32(19):3938-3948.

35. Vizzardelli C, et al. Effects of dexamethazone on LPSinduced activationand migration of mouse dendritic cells revealed by a genome-wide transcriptional analysis. Eur Immunol. 2006;36(6):1504-1515.

36. Recalcati S, et al. Differential regulation of iron homeostasis during human macrophage polarized activation. Eur I Immunol. 2010;40(3):824-835.

37. Edwards JP, Zhang X, Frauwirth KA, Mosser DM. Biochemical and functional characterization of three activated macrophage populations. J Lenkoc Biol. 2006;80(6):1298-1307.

38. Goetz DH, Holmes MA, Borregaard N, Bluhm ME, Raymond KN, Strong RK. The neutrophil lipocalin NGAL is a bacteriostatic agent that interferes with siderophore-mediated iron acquisition. Mol Cell. 2002;10(5):1033-1043.
39. Yang J, et al. An iron delivery pathway mediated by a lipocalin. Mol Cell. 2002;10(5):1045-1056

40. Devireddy LR, Gazin C, Zhu X, Green MR. A cellsurface receptor for lipocalin $24 \mathrm{p} 3$ selectively mediates apoptosis and iron uptake. Cell. 2005; 123(7):1293-1305.

41. Bachman MA, Miller VL, Weiser JN. Mucosal lipocalin 2 has pro-inflammatory and iron-sequestering effects in response to bacterial enterobactin. PLoS Pathog. 2009;5(10):e1000622.

42. Srinivasan G, et al. Lipocalin 2 deficiency dysregulates iron homeostasis and exacerbates endotoxininduced sepsis. J Immunol. 2012;189(4):1911-1919.

43. Charlson ES, et al. Topographical continuity of bacterial populations in the healthy human respiratory tract. Am J Respir Crit Care Med. 2011;184(8):957-963.

44. Bachman MA, et al. Klebsiella pneumoniae yersiniabactin promotes respiratory tract infection through evasion of lipocalin 2. Infect Immun. 2011; 79(8):3309-3316

45. Zhang X, Majlessi L, Deriaud E, Leclerc C, Lo-Man R. Coactivation of Syk kinase and MyD88 adaptor protein pathways by bacteria promotes regulatory properties of neutrophils. Immunity. 2009; 31(5):761-771.

46. Jung M, et al. Infusion of IL-10-expressing cells protects against renal ischemia through induction of lipocalin-2. Kidney Int. 2012;81(10):969-982.

47. Clausen BE, Burkhardt C, Reith W, Renkawitz R, Forster I. Conditional gene targeting in macrophages and granulocytes using LysMcre mice. Transgenic Res. 1999;8(4):265-277.

48. Alonzi T, Maritano D, Gorgoni B, Rizzuto G, Libert C, Poli V. Essential role of STAT3 in the control of the acute-phase response as revealed by inducible gene inactivation [correction of activation] in the liver. Mol Cell Biol. 2001;21(5):1621-1632.

49. Rijneveld AW, Florquin S, Branger J, Speelman P, Van Deventer SJ, van der Poll T. TNF-alpha compensates for the impaired host defense of IL-1 type I receptor-deficient mice during pneumococcal pneumonia. J Immunol. 2001;167(9):5240-5246.

50. Knapp S, et al. Activation of neutrophils and inhibition of the proinflammatory cytokine response by endogenous granulocyte colony-stimulating factor in murine pneumococcal pneumonia. J Infect Dis. 2004;189(8):1506-1515

51. Knapp S, et al. Toll-like receptor 2 plays a role in the early inflammatory response to murine pneumococcal pneumonia but does not contribute to antibacterial defense. J Immunol. 2004;172(5):3132-3138.

52. Zhang X, Goncalves R, Mosser DM. The isolation and characterization of murine macrophages. Curr Protoc Immunol. 2008; Chapter 14:Unit14.1.

53. Raoust E, Balloy V, Garcia-Verdugo I, Touqui L, Ramphal R, Chignard M. Pseudomonas aeruginosa LPS or flagellin are sufficient to activate TLR-dependent signaling in murine alveolar macrophages and airway epithelial cells. PLoS One. 2009;4(10):e7259.

54. Draing C, et al. Comparison of lipoteichoic acid from different serotypes of Streptococcus pneumoniae. J Biol Chem. 2006;281(45):33849-33859.

55. Morath S, Geyer A, Hartung T. Structure-function relationship of cytokine induction by lipoteichoic acid from Staphylococcus aureus. J Exp Med. 2001; 193(3):393-397.

56. Brightbill HD, Plevy SE, Modlin RL, Smale ST. A prominent role for $\mathrm{Sp} 1$ during lipopolysaccharidemediated induction of the IL-10 promoter in macrophages. J Immunol. 2000;164(4):1940-1951. 